\title{
Prevalencia de sobrepeso y obesidad en niños escolares
}

\author{
Emanuel de J. Torres-González, ${ }^{1}$ Rosa G. Zamarripa-Jáuregui, ${ }^{1}$ José M. Carrillo-Martínez, ${ }^{1}$ \\ Fernando Guerrero-Romero ${ }^{2}$ y Gerardo Martínez-Aguilar 2* \\ 'Universidad Juárez del Estado de Durango, Facultad de Medicina y Nutrición; ²Instituto Mexicano del Seguro Social, Unidad de Investigación
} Biomédica. Durango, México

\section{Resumen}

Introducción: La obesidad infantil es un reto de salud pública. Entre 1999 y 2012, en México la prevalencia de sobrepeso y obesidad (SO) en niños escolares pasó de 25.5 a 32 \%. Objetivo: Reportar la prevalencia actual de SO en niños escolares del municipio de Durango, México. Método: Encuesta transversal realizada entre enero de 2017 y diciembre de 2018. Se incluyeron 24600 niños de seis a 11 años, de 138 escuelas del municipio de Durango. Se utilizaron los valores de referencia del índice de masa corporal establecidos por la Organización Mundial de la Salud para determinar la presencia de SO. Resultados: La prevalencia de sobrepeso fue de $19.7 \%$, la de obesidad de $16 \%$ y la de SO de $35.7 \%$. En el grupo de seis años se encontró una prevalencia de SO de $25.4 \%$ y en el de 11 años, de $41.1 \%$. Conclusiones: La prevalencia de SO en niños de seis a 11 años del municipio de Durango es más elevada que la reportada en la encuesta nacional por entidad federativa en 2012 y la nacional en 2016; se observó tendencia al incremento conforme aumenta la edad.

PALABRAS CLAVE: Obesidad. Sobrepeso. Escolares.

\section{Prevalence of overweight and obesity in school-age children}

\section{Abstract}

Introduction: Childhood obesity is a public health challenge. Between 1999 and 2012, the prevalence in Mexico of overweight and obesity in schoolchildren went from 25.5 to $32 \%$. Objective: To report current prevalence of overweight and obesity in schoolchildren from the municipality of Durango, Mexico. Method: Cross-sectional survey conducted between January 2017 and December 2018. A total of 24,600 children aged between six and 11 years from 138 schools of the municipality of Durango were included. The body mass index reference values established by the World Health Organization were used to determine the presence of overweight and obesity. Results: The prevalence of overweight was $19.7 \%$, of obesity, $16 \%$, and of overweight and obesity combined, $35.7 \%$. In the six-year-old group, a prevalence of overweight-obesity of $25.4 \%$ was found, and in the 11-year-old group, $41.1 \%$. Conclusions: The prevalence of overweight-obesity in children aged from 6 to 11 years in the municipality of Durango is higher than those reported in the national survey by states in 2012 and in the 2016 national survey; prevalence showed a tendency to increase with age.

KEY WORDS: Obesity. Overweight. School-age children.

Correspondencia:

*Gerardo Martínez-Aguilar

E-mail: uimec@yahoo.es
Fecha de recepción: 22-11-2019

Fecha de aceptación: 19-02-2020

DOI: $10.24875 / G M M .20005642$
Gac Med Mex. 2020;156:184-187

Disponible en PubMed

www.gacetamedicademexico.com

0016-3813/৫ 2020 Academia Nacional de Medicina de México, A.C. Publicado por Permanyer. Este es un artículo open access bajo la licencia CC BY-NC-ND (http://creativecommons.org/licenses/by-nc-nd/4.0/). 


\section{Introducción}

Si bien la prevalencia de sobrepeso y obesidad (SO) en niños se ha incrementado durante los últimos 50 años, tanto en los países en desarrollo como en los desarrollados, la tasa de incremento de la obesidad infantil es hasta $30 \%$ más alta en los países con ingreso económico bajo o moderado que en aquellos con ingreso alto. ${ }^{1}$ Lo anterior ha llevado a considerar a la obesidad infantil como un problema global de salud pública emergente, con consecuencias sociales y económicas aún no determinadas.

Los niños con SO por lo general continúan con obesidad durante su vida y tienen mayor probabilidad de presentar enfermedades no transmisibles tales como diabetes, alteraciones cardiovasculares, dislipidemia, problemas ortopédicos, neurológicos, pulmonares y hepáticos. . $^{2,3}$

En México, la prevalencia de SO en niños escolares mostró un incremento significativo entre 1999 y 2012 : pasó de 25.5 a $32 \%{ }^{4}$

Los resultados de la Encuesta Nacional de Salud y Nutrición (ENSANUT) $2012^{5}$ mostraron que en el estado de Durango la prevalencia de SO en niños de cinco a 11 años fue de $33.8 \%$, mayor en los varones $(37.5 \%)$ que en las niñas $(29.8 \%)$. ENSANUT de Medio Camino de $2016^{6}$ reportó una prevalencia nacional de SO de $32.8 \%$ en niñas y $33.7 \%$ en niños; en el norte de México, la prevalencia de SO en niños de cinco a 11 años fue de $29.5 \%$.

El objetivo de este estudio fue determinar la prevalencia de SO en niños que asisten a escuelas primarias del municipio de Durango.

\section{Método}

Previa aprobación del estudio por el Comité de Ética en Investigación del Instituto Mexicano del Seguro Social (2016-785-103) y después de la obtención del consentimiento informado de los padres de todos los participantes, se desarrolló un estudio transversal descriptivo en colaboración con el Sistema Nacional para el Desarrollo Integral de la Familia de Durango, a través del programa Salud Integral que el gobierno del estado realiza en las escuelas primarias, y que entre sus acciones incluye la valoración nutricional de los niños inscritos en las escuelas primarias del sistema público.

Entre enero de 2017 y diciembre de 2018 se incluyeron todos los niños de seis a 11 años incorporados en el programa Salud Integral para ese periodo, inscritos en 138 (68.3\%) escuelas de un total de 202 localizadas en el municipio de Durango.

Profesionales en nutrición previamente capacitados determinaron el peso y la talla utilizando básculas (Beurer, modelo MS 50) y estadímetros portátiles (Seca, modelo 213).

El diagnóstico de SO se estableció de acuerdo con el índice de masa corporal (IMC) conforme las tablas de referencia de la Organización Mundial de la Salud (valores Z de IMC para la edad de cinco a 19 años). ${ }^{7}$ De acuerdo con el número de habitantes, las localidades se clasificaron en urbanas (más de 2500 habitantes) y rurales (menos de 2500 habitantes).

La información se recolectó en hojas de cálculo de Excel y se analizó con el programa estadístico SPSS versión 15.

\section{Resultados}

Al estudio se incorporaron 24600 niños, $77.1 \%$ procedía de localidades urbanas y $22.9 \%$ de localidades rurales. La prevalencia de SO fue de $35.7 \%$ ( $19.7 \%$ de sobrepeso y $16.0 \%$ de obesidad); en escuelas de localidades urbanas la prevalencia fue de $36.7 \%$ y en las rurales, de $31.7 \%$.

En las localidades urbanas, la prevalencia de sobrepeso fue mayor en las niñas $(21.2 \%)$ que en los niños (19.1\%), mientras que la de obesidad fue mayor en los niños (19.2 \%) que en las niñas (14.3\%). En el medio rural se observó un patrón similar, con mayor sobrepeso en niñas (18.9\%) que en niños (17.7\%) y mayor prevalencia de obesidad en varones (15.0\%) que en mujeres (11.7 \%) (Tabla 1).

Cuando se analizaron los resultados de acuerdo con la edad, la prevalencia de SO fue de $25.4 \%$ en los niños de seis años y de $41.1 \%$ en los de 11 años (Tabla 2).

\section{Discusión}

La prevalencia de SO en niños de seis a 11 años del municipio de Durango fue de $35.7 \%$, $1.9 \%$ (1.2\% de sobrepeso y $0.7 \%$ de obesidad) mayor a la reportada en ENSANUT $2012^{5}$ (Tabla 3).

De acuerdo con los datos de ENSANUT de Medio Camino de 2016, la prevalencia nacional de SO fue de $33.2 \%{ }^{6}$ Dado que la encuesta no proporcionó resultados por entidad federativa, las prevalencias registradas en nuestro estudio se compararon con las reportadas en ENSANUT 2012. ${ }^{5}$ Según el sexo, la 
Tabla 1. Prevalencia de sobrepeso y obesidad por tipo de localidad y sexo

\begin{tabular}{|c|c|c|c|c|c|c|c|c|c|c|c|c|c|c|c|c|c|c|}
\hline \multirow[t]{3}{*}{ Localidad } & \multicolumn{9}{|c|}{ Niñas } & \multicolumn{9}{|c|}{ Niños } \\
\hline & \multicolumn{2}{|c|}{ Peso normal } & \multicolumn{2}{|c|}{ Sobrepeso } & \multicolumn{2}{|c|}{ Obesidad } & \multicolumn{2}{|c|}{ so } & \multirow{2}{*}{$\begin{array}{c}\text { Total } \\
\mathbf{n}\end{array}$} & \multicolumn{2}{|c|}{ Peso normal } & \multicolumn{2}{|c|}{ Sobrepeso } & \multicolumn{2}{|c|}{ Obesidad } & \multicolumn{2}{|c|}{ so } & \multirow{2}{*}{\begin{tabular}{|c|} 
Total \\
$\mathbf{n}$
\end{tabular}} \\
\hline & $\mathrm{n}$ & $\%$ & $\mathrm{n}$ & $\%$ & $\mathrm{n}$ & $\%$ & $\mathrm{n}$ & $\%$ & & $\mathrm{n}$ & $\%$ & $\mathrm{n}$ & $\%$ & $\mathrm{n}$ & $\%$ & $\mathrm{n}$ & $\%$ & \\
\hline Urbana & 6147 & 64.6 & 2014 & 21.2 & 1358 & 14.3 & 3372 & 35.4 & 9519 & 5829 & 61.7 & 1803 & 19.1 & 1813 & 19.2 & 3616 & 38.3 & 9445 \\
\hline Rural & 1977 & 69.4 & 537 & 18.9 & 334 & 11.7 & 871 & 30.6 & 2848 & 1876 & 67.3 & 493 & 17.7 & 419 & 15 & 912 & 32.7 & 2788 \\
\hline Total & 8124 & 65.7 & 2551 & 20.6 & 1692 & 13.7 & 4243 & 34.3 & 12367 & 7705 & 63 & 2296 & 18.8 & 2232 & 18.2 & 4528 & 37 & 12233 \\
\hline
\end{tabular}

Tabla 2. Prevalencia de sobrepeso y obesidad de acuerdo con la edad

\begin{tabular}{|c|c|c|c|c|c|c|c|c|c|c|c|c|c|c|c|c|c|c|}
\hline \multirow{3}{*}{$\begin{array}{l}\text { Edad } \\
\text { (años) }\end{array}$} & \multicolumn{9}{|c|}{ Niñas } & \multicolumn{9}{|c|}{ Niños } \\
\hline & \multicolumn{2}{|c|}{ Peso normal } & \multicolumn{2}{|c|}{ Sobrepeso } & \multicolumn{2}{|c|}{ Obesidad } & \multicolumn{2}{|c|}{ so } & \multirow{2}{*}{$\frac{\text { Total }}{n}$} & \multicolumn{2}{|c|}{ Peso normal } & \multicolumn{2}{|c|}{ Sobrepeso } & \multicolumn{2}{|c|}{ Obesidad } & \multicolumn{2}{|c|}{ so } & \multirow{2}{*}{$\frac{\text { Total }}{n}$} \\
\hline & $\mathrm{n}$ & $\%$ & $\mathrm{n}$ & $\%$ & $\mathrm{n}$ & $\%$ & $\mathrm{n}$ & $\%$ & & $\mathrm{n}$ & $\%$ & $\mathrm{n}$ & $\%$ & $\mathrm{n}$ & $\%$ & $\mathrm{n}$ & $\%$ & \\
\hline 6 & 1640 & 76.1 & 334 & 15.5 & 180 & 8.4 & 514 & 23.9 & 2154 & 1606 & 73.1 & 353 & 16.1 & 237 & 10.8 & 590 & 26.9 & 2196 \\
\hline 7 & 1417 & 69.0 & 372 & 18.1 & 266 & 12.9 & 638 & 31.0 & 2055 & 1437 & 66.7 & 376 & 17.4 & 344 & 15.9 & 720 & 33.3 & 2157 \\
\hline 8 & 1349 & 62.6 & 475 & 22.1 & 330 & 15.3 & 805 & 37.4 & 2154 & 1258 & 63.6 & 346 & 17.5 & 374 & 18.9 & 720 & 36.4 & 1978 \\
\hline 9 & 1338 & 63.9 & 468 & 22.3 & 290 & 13.8 & 758 & 36.1 & 2096 & 1194 & 59.4 & 379 & 18.9 & 435 & 21.7 & 814 & 40.6 & 2008 \\
\hline 10 & 1270 & 61.8 & 463 & 22.5 & 323 & 15.7 & 786 & 38.2 & 2056 & 1153 & 55.9 & 444 & 21.5 & 467 & 22.6 & 911 & 44.1 & 2064 \\
\hline 11 & 1110 & 59.9 & 439 & 23.7 & 303 & 16.4 & 742 & 40.1 & 1852 & 1057 & 57.8 & 398 & 21.7 & 375 & 20.5 & 773 & 42.2 & 1830 \\
\hline
\end{tabular}

Tabla 3. Prevalencia de sobrepeso y obesidad en Durango de acuerdo con ENSANUT 2012 y al presente estudio

\begin{tabular}{|c|c|c|c|c|c|c|}
\hline \multicolumn{2}{|c|}{ Niñas } & \multicolumn{3}{c|}{ Niños } \\
\cline { 2 - 7 } & Sobrepeso & Obesidad & So & Sobrepeso & Obesidad & So \\
\hline $2012^{*}$ & $15.9(10-223.9)$ & $13.9(9.2-20.3)$ & $29.8(21.9-39.0)$ & $20.8(15.7-27.0)$ & $16.7(11.0-24.4)$ & $37.5(30.2-45.4)$ \\
\hline $2018^{* *}$ & $20.6(19.8-21.3)$ & $13.7(13.0-14.3)$ & $34.3(33.4-35.1)$ & $18.8(18.1-19.4)$ & $18.2(17.5-18.8)$ & $37.0(36.1-37.8)$ \\
\hline
\end{tabular}

Los valores representan la prevalencia y los intervalos de confianza de $95 \%$. SO = sobrepeso + obesidad.

*Datos de ENSANUT $2012^{5 * *}$ Datos de este estudio.

prevalencia de SO tuvo un incremento de $4.5 \%$ en las niñas, mientras que en los niños disminuyó en $0.5 \%$.

El incremento de la prevalencia de SO en las niñas estuvo determinado por el incremento importante de la prevalencia de sobrepeso, que pasó de $15.9 \%$ a $20.6 \%$ en seis años. En los niños, el sobrepeso disminuyó $2 \%$ : de $20.8 \%$ pasó a $18.8 \%$. La prevalencia de obesidad en las niñas disminuyó en $0.2 \%$, mientras que en los niños se incrementó en $1.5 \%$.

Respecto al tipo de localidad, la prevalencia de SO se incrementó en $1.2 \%$ (0.6\% de sobrepeso y $0.6 \%$ de obesidad) en las localidades urbanas, mientras que en las rurales el incremento fue de $1.7 \%$ (1.9\% de sobrepeso y $0.2 \%$ de obesidad).

Al analizar los datos de acuerdo con la edad, la prevalencia de SO fue menor a los seis años que a los 11 (25.4 y $41.1 \%)$. Cuando se desagregó la información de acuerdo con el sexo se observó la misma tendencia. Este resultado sugiere que, al momento de su ingreso a la escuela primaria, una cuarta parte de los niños presenta problemas de sobrepeso u obesidad, probablemente relacionados con el estilo de vida en el entorno familiar y factores genéticos (genoma y 
microbioma intestinal) y con el hecho de que en las escuelas existen entornos adversos que favorecen el desarrollo de SO.

En comparación con ENSANUT 2006, ${ }^{8}$ que al igual que nuestro estudio proporcionó resultados por grupos de edad, destacó que la prevalencia de SO en los niños de 11 años (32.5\%) fue mayor que en los de cinco $(17.7 \%$ años. De forma similar, la prevalencia de SO en niñas de 11 años fue mayor (29.5\%) que la de niñas de cinco años (21.2\%).

En comparación con ENSANUT 2012, ${ }^{5}$ ENSANUT $2006^{7}$ reportó una disminución leve en la prevalencia de sobrepeso en niñas (16.5\% versus $15.9 \%$ ), por lo que la prevalencia de $20.6 \%$ documentada en el presente estudio es la más alta observada en los últimos 12 años en la entidad.

El incremento de la prevalencia de sobrepeso en niñas debe considerarse como una advertencia, ya que el riesgo de desarrollar obesidad y las comorbilidades que se le asocian aumentan si esa condición continúa hasta la adolescencia, etapa en la que hay cambios en la composición corporal relacionados con la pubertad (caracterizada por disminución de la sensibilidad a la insulina que promueve el incremento de grasa corporal), aunados a la disminución de la actividad física regular e incremento del consumo de alimentos con mayor contenido calórico. $^{9}$

Aun cuando el problema que representa la elevada prevalencia de SO está bien documentado en los escolares y se han establecido diversas intervenciones de educación nutricional y recomendaciones de actividad física, estas no han sido eficaces para detenerlo, lo que sugiere que las políticas públicas en este rubro requieren modificaciones sustanciales..$^{10,11}$

El acceso al total de la muestra proporcionó solidez y confiabilidad a los resultados, como se demostró con los reducidos intervalos de confianza, considerablemente menores que los obtenidos en las ENSANUT del 2006 y 2012.,5 Una debilidad de nuestro estudio es que la prevalencia registrada pudiera no representar la de otras poblaciones rurales del estado, debido a la cercanía con la ciudad de Durango.

\section{Agradecimientos}

Agradecemos sinceramente el apoyo de Elvira Barrantes de Aispuro, presidente del Sistema Nacional para el Desarrollo Integral de la Familia de Durango, y de Rocío Manzano Chaídez, directora del Sistema Estatal para el Desarrollo Integral de la Familia, así como del personal adscrito al programa Salud Integral del gobierno del estado de Durango, sin cuya colaboración este trabajo no hubiera sido posible.

\section{Conflicto de intereses}

Ninguno.

\section{Fuentes de Financiamiento}

Para esta investigación se recibieron los financiamientos FIS/IMSS/PROT/1762 y FIS/IMSS/PROT/1762 del Consejo de Ciencia y Tecnología del Estado de Durango, y FIS/IMSS/PROT/G17-21727 del Instituto Mexicano del Seguro Social.

\section{Responsabilidades éticas}

Los autores declaran que para esta investigación no se realizaron experimentos en seres humanos ni en animales.

Confidencialidad de los datos. Los autores declaran que siguieron los protocolos de su centro de trabajo sobre la publicación de datos de terceros.

Derecho a la privacidad y consentimiento informado. Los autores declaran que en este artículo no aparecen datos de pacientes.

\section{Bibliografía}

1. Gluckman P, Nishtar S, Armstrong T. Ending childhood obesity: a multidimensional challenge. Lancet. 2015;385:1048-1050.

2. World Health Organization. Obesity and overweight. Suiza: WHO; 2018.

3. World Health Organization. Diet, nutrition and the prevention of chronic diseases. World Health Organ Tech Rep Ser. 2009:916:1-149.

4. Hernández-Cordero S, Cuevas-Nasu L, Morán-Ruán MC, Méndez-Gómez I, Ávila-Arcos MA, Rivera-Dommarco JA. Overweight and obesity in Mexican children and adolescents during the last 25 years. Nutr Diabetes. 2017:7:e247.

5. Encuesta Nacional de Salud y Nutrición 2012. Resultados por entidad federativa. México: Instituto Nacional de Salud Pública; 2013.

6. Encuesta Nacional de Salud y Nutrición de Medio Camino 2016. Informe final de resultados. México: Instituto Nacional de Salud Pública, 2016.

7. World Health Organization [sitio web]. Growth references 5-19 years. Suiza: WHO; 2018.

8. Instituto Nacional de Salud Pública. Encuesta Nacional de Salud y Nutrición 2006. México: INSP; 2006.

9. Todd AS, Street SJ, Ziviani J, Byrne NM, Hills AP. Overweight and obese adolescent girls: the importance of promoting sensible eating and activity behaviors from the start of the adolescent period. Int $\mathrm{J}$ Environ Res Public Health. 2015;12:2306-2329.

10. Wang $Y$, Cai L, Wu Y, Wilson RF, Weston C, Fawole O, et al. What childhood obesity prevention programmes work? A systematic review and meta-analysis. Obes Rev. 2015;16:547-565.

11. Wolfenden L, Wyse R, Nichols M, Allender S, Millar L, McElduff P. A systematic review and meta-analysis of whole of community interventions to prevent excessive population weight gain. Prev Med. 2014;62:193200. 